\title{
La educación constitucional: caracterización general y recepción en el sistema constitucional español
}

\author{
Constitutional Education: general \\ characterisation and its reception in the \\ Spanish Constitutional System
}

\section{Francisco Manuel García Costa}

Prof. Titular de Derecho Constitucional

Departamento de Fundamentos de Orden Jurídico y Constitucional

Universidad de Murcia (España)

E-mail: fmgarcia@um.es

\begin{abstract}
Resumen: En el presente trabajo se analiza si el derecho fundamental a la educación del artículo 27.1 C.E. y los fines de la acción educativa de los poderes públicos recogidos en el artículo 27.2. C.E. exigirían o, al menos, posibilitarían la inserción en el sistema educativo español de la Educación Constitucional, es decir, la formación en la Constitución en los niveles obligatorios del sistema educativo. Para ello, examinamos en primer lugar, en clave de teoría general, algunas de las cuestiones fundamentales de la Educación Constitucional: la caracterización de este nuevo tipo de educación; su diferenciación de otras disciplinas similares cuyo objeto no es la exposición de la Constitución, sino la de la convivencia social; su fundamentación, como instrumento de una suerte de defensa paidética de la Constitución y del orden constitucional; y, por último, su condición, en tanto en cuanto la Educación Constitucional es una educación
\end{abstract}


para los derechos humanos y para la igualdad de género, de instrumento para la realización de las metas del ODS nº 4 . A partir de estas cuestiones generales, concluimos que nuestra Constitución, a pesar de que no declara expresamente la obligatoriedad de su enseñanza en la Educación Primaria y Secundaria, la permite y que incluso cabría plantearse legítimamente la cuestión de la existencia implícita de esta obligatoriedad deducida de los mencionados artículos 27.1 C.E., 27.2 C.E. en relación con los artículos 27.5 C.E., 27.3 C.E. y 9.2 C.E.

Palabras clave: Educación constitucional, educación en valores sociales y cívicos, objetivos de desarrollo sostenible.

Abstract: This paper analyses whether the fundamental right to education in article 27.1 of the Spanish Constitution (CE) and the aims of the educational action of the public authorities set out in article 27.2 of the CE would require or, at least, make possible the insertion in the Spanish educational system a subject of Constitutional Education, that is, training in the Constitution at the compulsory levels of the education system. To this end, we first examine, in terms of general theory, some of the fundamental issues of Constitutional Education: the characterisation of this new type of education; its differentiation from other similar disciplines whose object is not the exposition of the Constitution, but that of social coexistence; its foundation, as an instrument of a kind of paid defense of the Constitution and the constitutional order; and, finally, its status, insofar as Constitutional Education is an education for human rights and gender equality, as an instrument for the achievement of the goals of SDG no. 4. On the basis of these general questions, we conclude that our Constitution, although it does not expressly declare the compulsory nature of its teaching in Primary and Secondary Education, allows it and that the question of the implicit existence of this compulsory nature deduced from the aforementioned articles 27.1 CE and 27.2 CE in relation to articles 27.5 CE, 27.3 CE and 9.2 CE could even be legitimately raised.

Keywords: Constitutional education, education in social and civic values, sustainable development goals. 
Sumario: 1. Hacia una caracterización de la Educación Constitucional. 2. Fundamento de la Educación Constitucional. 3. La Educación Constitucional en el ámbito del ODS nº4. 4. La Educación Constitucional en el sistema constitucional español. 4.1. La Educación Constitucional en la Constitución de 1978. 4.2. La Educación Constitucional en la legislación en materia educativa. 5. Conclusiones. 6. Bibliografía.

"El plan general de enseñanza será uniforme en todo el Reino, debiendo explicarse la Constitución política de la Monarquía en todas las Universidades y establecimientos literarios donde se enseñen las ciencias eclesiásticas y políticas"

Artículo 368 de la Constitución de Cádiz

“Todos los alumnos recibirán un ejemplar de la Constitución al terminar sus deberes escolares"

Artículo 148 de la Constitución de Weimar

\section{Hacia una caracterización de la Educación Constitucional}

Se entiende por Educación Constitucional la enseñanza de la Constitución de un concreto Estado en los niveles obligatorios de su sistema educativo. Digamos primero, siguiendo un método muy tradicional, quid non sit e intentemos después caracterizarla.

(i) En primer lugar, la Educación Constitucional no es la enseñanza en los niveles obligatorios del sistema educativo de la estructura social, de la vida en comunidad o de la convivencia social, sino de la Constitución, sin que sea posible la existencia de contenidos de la Educación Constitucional que no sean los propios del texto de la Constitución, de manera que la formación en las realidades sociales y política de un país lo será en la medida en que estas se encuentren directa e inmediatamente vinculadas a la explicación de la Constitución.

Consiguientemente, la Educación Constitucional ha de distinguirse de materias afines que, por venir centradas en el estudio de la sociedad o de la convivencia en sociedad, pueden referirse a la Constitución, si bien su objeto no es estrictamente la Constitución y, por ello, su finalidad no es la formación e instrucción en la Constitución, sino aprender a vivir en común. Es por ello que la Educación Constitucional se distingue en su objeto de disciplinas similares como las de "Educación para la Ciudadanía y los Derechos Humanos", "Educación en Valores Cívicos y Éticos” y otras tantas cuyos contenidos no 
se corresponden íntegramente con la explicación de la Constitución. Consiguientemente, la "Educación Constitucional" comprende la explicación de todos los valores y reglas de la Constitución, mientras que la "Educación en valores cívicos", la "Educación para la ciudadanía" y otras disciplinas afines comprenden la explicación de contenidos externos a la Constitución y de todos o parte -lo que es tendencia mayoritaria- de los valores y reglas de la Constitución.

En el entendido de que la Educación Constitucional versa sobre los valores y reglas constitucionales, la formación en derechos humanos, en derechos fundamentales y libertades públicas, en igualdad y no discriminación, en respeto a la democracia y en respeto al Estado de Derecho deben ser consideradas como contenidos de la Educación Constitucional, en tanto en cuanto los anteriores no solo son elementos concretos y específicos de toda Constitución, sino también los valores fundamentales del movimiento constitucional y del Estado constitucional. Item más: la garantía de los derechos, el principio de igualdad con la correlativa prohibición de discriminación, el Estado de Derecho y la democracia representativa son aportaciones del movimiento constitucional, apareciendo de manera definitiva en la Historia vinculados, precisamente, al constitucionalismo. Sobre este punto volveremos en particular en el epígrafe 3 de este trabajo al tratar de la relación entre la Educación Constitucional y el ODS nº 4.

(ii) La Educación Constitucional no es la enseñanza de la Constitución en los niveles no obligatorios del sistema educativo. Consiguientemente, se distingue del Derecho Constitucional en tanto en cuanto este, como es bien sabido, es la Ciencia que estudia desde los Elementi di diritto costituzionale democratico de Compagnoni di Luzo de 1797las constituciones en el ámbito de las alturas venerables de las Facultades de Derecho y, consiguientemente, más allá de los niveles obligatorios del sistema educativo. El Derecho Constitucional, entendido en los términos del gran Ramón de Salas como "Ciencia de la Libertad", consiste en una disciplina jurídica cuya función no es la convencer, sino la de formar; de ahí que su conocimiento sea uno de los elementos de la virtud intelectual o cualidad mental de la Jurisprudencia, que es requerible tan solo en el profesional del Derecho. Añádase al argumento anterior que la libertad de cátedra en su dimensión positiva no presenta el mismo grado de intensidad en la educación universitaria y en las educaciones primaria y secundaria, pues la libertad del docente en la determinación autónoma del contenido y del método de transmisión del conocimiento es máxima en el 
nivel universitario, mientras que su intensidad disminuye en los otros dos niveles educativos, pues en estos son los planes de estudios establecidos por la autoridad competente, y no el propio profesor, los que determinan cuál haya de ser el contenido mínimo de la enseñanza, y son también estas autoridades las que establecen cuál es el elenco de medios pedagógicos entre los que puede optar el profesor. Consiguientemente, la explicación de la Constitución en las Universidades en que consiste el Derecho constitucional está garantizada por la libertad de cátedra en su grado máximo, mientras que la explicación de la Constitución en los Colegios e Institutos en que consiste la Educación Constitucional está, por el contrario, garantizada por la libertad de cátedra en un grado limitado. Como conclusión, podemos afirmar que, aunque exista coincidencia en el objeto material -en el estudio de la Constitución-, la Educación Constitucional no puede ser identificada con el Derecho Constitucional.

(iii) Una vez que sabemos que la Educación Constitucional no puede ser Derecho Constitucional porque su destinatario es el alumnado de los ciclos obligatorios del sistema educativo, ni tampoco puede ser una educación cívica fundamentada en las reglas y valores de la Constitución, estamos en disposición de poder delimitarla positivamente.

-En primer lugar, la Educación Constitucional se define por su objeto singular: la explicación de la Constitución.

-En segundo lugar, la Educación Constitucional se define por su destinatario: el alumnado de los niveles obligatorios del sistema educativo.

La especificación, consecuentemente, de cuáles son los contenidos concretos dentro de la Constitución que podrán ser objeto de la Educación Constitucional y del tratamiento de cada uno de los mismos en atención a su obligatoriedad en el sistema educativo vendrá determinada en cada ordenamiento jurídico en función de la regulación de la materia educativa en las respectivas constituciones y, en particular, del reconocimiento en las mismas de la propia obligación de estudiar la Constitución; del derecho a la educación como derecho social prestacional; del reconocimiento de las libertades educativas (la libertad de cátedra con relación a su intensidad en los términos que hemos adelantado anteriormente, la libertad de creación de centros, el derecho de los padres para que sus hijos reciban la formación religiosa y moral que esté de acuerdo con sus propias 
convicciones y la autonomía universitaria); de los principios de la política educativa; de los medios de la misma; o, en fin, de los fines y los contenidos de la acción educativa de los poderes públicos. En particular, sobre los contenidos concretos y la recepción de la Educación Constitucional en el sistema constitucional español volveremos en el punto 4 de este trabajo.

(iv) De la misma manera que las materias "Educación en Ciudadanía" (artículo 347 de la Constitución de Ecuador), "Educación Cívica" (artículos 31 y 41 de la Constitución de los Estados Unidos Mexicanos), "Educación Ciudadana” (artículo 102 de la Constitución de la República Bolivariana de Venezuela), "Educación para la Ciudadanía y los Derechos Humanos" (art. 18.3 de la L.O. 2/2006, de 3 de mayo, de Educación), “Educación en Valores Sociales y Cívicos” (art. 8 del Real Decreto 126/2014, de 28 de febrero, por el que se currículo básico de la Educación Primaria) y "Educación en Valores Cívicos y Éticos” (la Ley Orgánica 3/2020, de 29 de diciembre, por la que se modifica la Ley Orgánica 2/2006, de 3 de mayo, de Educación) han tenido reconocimiento por parte de las constituciones, de la legislación educativa y de la doctrina científica, también el estudio de la Constitución del respectivo estado en los niveles obligatorios del sistema constitucional, es decir, la Educación Constitucional, ha sido declarado obligatorio en ciertas constituciones, tanto históricas -la Constitución de Cádiz de 1812 y la Constitución de Weimar de 1919-, como actuales, todas ellas de estados iberoamericanos: así, el artículo 41 de la Constitución de Colombia de $1991^{80}$; el artículo 60 de la Constitución de El Salvador de 1983 ${ }^{81}$; el artículo 72 de la Constitución de Guatemala de $1985^{82}$; el artículo 168 de la Constitución de Honduras ${ }^{83}$; el artículo 117 de la Constitución de Nicaragua de $1987^{84}$; el artículo 18 de la Constitución de Paraguay de

\footnotetext{
${ }^{80} \mathrm{Su}$ tenor literal es el siguiente: "En todas las instituciones de educación, oficiales o privadas, serán obligatorios el estudio de la Constitución y la Instrucción Cívica. Así mismo se fomentarán prácticas democráticas para el aprendizaje de los principios y valores de la participación ciudadana. El Estado divulgará la Constitución". Las constituciones examinadas han sido consultadas on line en la página web del Proyecto Constitute https://www.constituteproject.org. Última consulta de todos los preceptos constitucionales que parecen citados en este trabajo: 18 de marzo de 2021.

${ }^{81}$ Su dicción literal es la siguiente: "En todos los centros docentes, públicos o privados, civiles o militares, será obligatoria la enseñanza de la historia nacional, el civismo, la moral, la Constitución de la República, los derechos humanos y la conservación de los recursos naturales [...] La historia nacional y la Constitución deberán ser enseñadas por los profesores salvadoreños"

${ }^{82}$ Este precepto dispone lo siguiente: "La enseñanza sistemática de la Constitución de la República y de los derechos humanos".

${ }^{83}$ Establece este precepto lo siguiente: "La enseñanza de la Constitución de la República, de la historia y geografía nacionales, es obligatoria y estará a cargo de profesionales hondureños".

${ }^{84} \mathrm{Su}$ tenor es el siguiente: "La educación [...] cultiva los valores propios del nuevo nicaragüense, de acuerdo con los principios establecidos en la presente Constitución, cuyo estudio deberá ser promovido".
} 
1992 ${ }^{85}$; el artículo 14 de la Constitución de Perú de 1993 ${ }^{86}$; y, por último, el artículo 63 de la Constitución de la República Dominicana de $2015^{87}$.

Sin embargo, la denominación que aquí acogemos, Educación Constitucional, no ha recibido, hasta el momento y en lo que se nos alcanza, carta de naturaleza, sea en el plano normativo, sea en el plano doctrinal.

\section{Fundamento de la Educación Constitucional}

Partiendo del entendido de que la Educación Constitucional es la formación en la Constitución de un concreto Estado en los niveles obligatorios de su sistema educativo, su fundamento ha de hallarse en los dos momentos en los que, precisamente, el estudio de la Constitución se convierte en obligatorio en el sistema educativo, que son los que representan el artículo 368 de la Constitución española de 1812 y los artículos 142 y 148 de la Constitución alemana de 1919.

Recordemos que el artículo 368 gaditano fue el que dispuso la obligación de explicar la Constitución de 1812 en todas las Universidades y establecimientos literarios donde se enseñasen las ciencias eclesiásticas y políticas, mientras que los artículos 142 y 148 alemanes fueron los primeros que establecieron el derecho a la educación ("El arte, la ciencia y su enseñanza son libres. El Estado los protege y participa en su mantenimiento" -art. 142-) y dispusieron que tal acción educativa tenía unos fines específicos ("en todas las escuelas se procurará la formación moral, la educación cívica y el perfeccionamiento personal y profesional; todo ello conforme al espíritu nacional y de reconciliación entre los pueblos") que se materializaron en unos contenidos concretos ("la educación cívica y la enseñanza profesional form[e]n parte de las disciplinas del plan escolar"-art. 148-). Coronando este artículo 148, se establecía la obligación de que todos los alumnos recibieran un ejemplar de la Constitución al terminar sus deberes escolares.

\footnotetext{
${ }^{85}$ Dispone lo siguiente: “A través del sistema educativo, se fomentará el estudio de la Constitución Nacional".

${ }^{86}$ La enseñanza de la Constitución y de los derechos humanos son obligatorias en todo el proceso educativo civil o militar".

87 "En todas las instituciones de educación pública y privada, serán obligatorias la instrucción en la formación social y cívica, la enseñanza de la Constitución”.
} 
(i) El primero de los momentos mencionados, Cádiz, nos recuerda que para los liberales gaditanos el proyecto de convivencia que se proponía en las constituciones suponía la sublimación y la apoteosis del desvelamiento progresivo de la idea de Libertad en la Historia y la realización del eterno motivo humano de que el poder de un hombre sobre otro fuera limitado y controlado. Siendo ello así, el instrumento para asentar las constituciones no podía ser la fuerza, sino una adecuada educación con la que, efectivamente, se penetrase en el ánimo y se subyugase la voluntad de los hombres a los que aquellos textos pretendían convertir en ciudadanos miembros de una Nación en la que residía esencialmente la Soberanía. Comparecía la Constitución como un nuevo orden político que había de ser asegurado, garantizado y defendido, primordialmente, a través de la pertinente y adecuada educación constitucional de los ciudadanos: la educación, pues, como un arma con la que defender las constituciones. Consiguientemente, la explicación de la Constitución en el sistema educativo tenía un fundamento que no era el de formar en las reglas de la vida en sociedad, sino el de convencer a la ciudadanía sobre las bondades de la Constitución, es decir, el de defender a la propia Constitución.

Efectivamente, la única manera de que los antiguos vasallos, convertidos en nuevos ciudadanos de la Nación Soberana, pudieran ser conscientes de todas las bondades y de los hermosos frutos de la Constitución era instruirlos y educarlos en ella -enseñándoles, principalmente, a leer y escribir- para que, al haber recibido todas las impresiones de la luz, se convirtiesen en amantes de la misma y la defendiesen incluso frente a Fernando VII. Agustín de Argüelles, con su habitual talento, lo explicaba perfectamente en el Discurso Preliminar a la Constitución de 1812: “el Estado, no menos que de soldados que le defiendan, necesita de ciudadanos que adiestren a la Nación y promuevan su felicidad con todo género de luces y de conocimientos" (Argüelles, 2011: 125).

La propia condición de existencia del artículo 368 residió no en cumplir con las exigencias del sistema educativo, sino con las de defender la Constitución. Item más. La propia configuración del sistema educativo (universalidad, uniformidad y gratuidad) fue exigencia de un nuevo sistema en el que la Constitución se defendía a través de la educación y, como sublimación de este planteamiento, a través de la específica formación en la propia Constitución. Consecuentemente, la instrucción en la Constitución se integró así como el más importante de los elementos de la defensa paidética de la Constitución 
entre los que figuraban, asimismo, los símbolos del poder (las solemnidades de la promulgación de la Constitución; su publicación y juramento en los pueblos, ejércitos y armada; las medidas extraordinarias que se aprobaron con ocasión de la misma; el sello de las Cortes) y las diferentes medidas de difusión y propagación de la Constitución (las medidas específicas para su impresión y circulación; los actos conmemorativos; los monumentos públicos; y el nuevo lenguaje constitucional) (García Costa, 2019: 346).

(ii) El segundo de los momentos mencionados, Weimar, nos lleva a conclusiones similares a las que hemos extraído de Cádiz. Como hemos adelantado, en la Constitución alemana de Weimar de 1919, con la que se inauguraba en la Europa continental el Estado Social, se reconoció por primera vez el derecho a la educación como derecho de naturaleza social y de contenido prestacional, el cual se satisfacía mediante una acción educativa de los poderes públicos que, además, estaba orientada a la consecución de ciertos fines y presentaba, asimismo, unos contenidos concretos, corolarios de aquellos, entre los que figuraba la "educación cívica". Su incorporación se produjo en el tiempo de la Constitución social en el que los poderes públicos empiezan a desarrollar una función transformadora de la sociedad orientada a lograr el pleno desarrollo de la persona humana en los órdenes económico y social mediante la supresión de los obstáculos que lo impidan; es decir -y en palabras de Garrorena- a "perseguir una transformación profunda de la sociedad que haga que la libertad, la justicia y la igualdad sean cada vez más reales y efectivas" (Garrorena, 2011: 69). Esta transformación social que asume el Estado se articula esencialmente mediante el reconocimiento y la garantía del derecho a la educación, por lo que este no solo comparece como uno más de los derechos sociales, sino como el vehículo privilegiado y excepcional para acometer la transformación de la sociedad desde y por el Estado. Siendo ello así y, en coherencia con estos planteamientos, la acción educativa de los poderes públicos se convirtió en instrumento primordial para conseguir los fines que las constituciones estiman dignos de alcanzar a través de la transmisión de una serie de valores y contenidos concretos.

Dada esta configuración de la educación en el Estado social, de la misma se desprende que el derecho a la educación no solo consiste en el acceso a las enseñanzas regladas, es decir, a las que componen el sistema educativo del Estado, sino a unas enseñanzas regladas que están orientadas a la consecución de ciertos fines mediante la transmisión de determinados valores y contenidos, consecuencia todo ello de la función 
transformadora de la sociedad propia del Estado social. Por ello, la mayor parte de las constituciones no regulan incondicionadamente el derecho a la educación de sus ciudadanos, sino que lo supeditan a la consecución de ciertos fines o lo estructuran conforme a ciertos contenidos, que son los propios del Estado Constitucional, tanto en el orden político como social: desde el respeto a los derechos humanos hasta la protección del medioambiente o de la salud.

Consiguientemente, la propia condición de existencia de la obligatoriedad de la enseñanza de la Constitución a través de la Educación Cívica residió, como en Cádiz, en la necesidad de conseguir transformar la sociedad hasta asentar plenamente los valores constitucionales en los que la misma se fundamentaba, necesidad a cuya satisfacción se enderezaba una educación que no estaba meramente orientada a la mejora del nivel de formación de la población, sino también a convencer sobre la legitimidad de los valores sobre los que se asentaba.

Cádiz y Weimar nos recuerdan que, en última instancia, la existencia de una formación en la Constitución en los niveles obligatorios no es una exigencia lógicamente derivada del sistema educativo, sino una exigencia de la defensa de la Constitución. Se trata del instrumento por antonomasia de la defensa paidética de la Constitución, es decir, de una defensa de la misma consistente en que, en el entendido de que solamente a través de la educación pueden implantarse y perpetuarse los regímenes constitucionales, la Constitución ha de ser propagada y difundida, siendo esta una tarea compartida por toda la sociedad y que utiliza como instrumento primordial al sistema educativo.

\section{La Educación Constitucional en el ámbito del ODS n4}

A partir del entendimiento de la Educación Constitucional como la instrucción y formación del alumnado de los niveles obligatorios del sistema educativo en las reglas y valores constitucionales, se colige que parte esencial de su contenido es la formación en derechos humanos y en igualdad de género; correlativamente, la formación en derechos humanos y en igualdad de género es, por definición, parte de la Educación Constitucional. Tal es la conclusión a la que hemos arribado en el punto anterior de este trabajo en la inteligencia de que los derechos fundamentales y el principio/derecho de igualdad son principios esenciales sobre los que se construyen el movimiento y la ideología 
constitucionales, así como su expresión institucionalizada que es el Estado Constitucional y su expresión normativa que son las constituciones. Por ello, enseñar y formar en derechos y en igualdad no es sino educar constitucionalmente: efectivamente, la instrucción en derechos humanos y en igualdad no es más que la instrucción en los preceptos de toda Constitución que regulan las libertades públicas y los derechos fundamentales (entre nosotros, los artículos del Título I C.E.), así como en los que regulan el principio/derecho a la igualdad formal (el artículo 14 C.E.) y el principio de igualdad material (así, el artículo 9.3 C.E.).

Siendo ello así, el Objetivo de Desarrollo Sostenible 4, "Garantizar una educación inclusiva, equitativa y de calidad y promover oportunidades de aprendizaje durante toda la vida para todos", se encuentra relacionado directamente con la Educación Constitucional en tanto en cuanto algunas de las metas de este ODS $n^{\circ} 4$ se alcanzan necesariamente con este tipo de educación. En efecto, dado que la meta 4.7 pretende "De aquí a 2030, asegurar que todos los alumnos adquieran los conocimientos teóricos y prácticos necesarios para promover el desarrollo sostenible, entre otras cosas mediante la educación para [...] los derechos humanos, la igualdad de género, la promoción de una cultura de paz y no violencia" ${ }^{\sharp 8}$, cabe entender que la Educación Constitucional, en su condición de educación para los derechos humanos y la igualdad de género, comparece como instrumento para la consecución de este ODS no 4 .

Por todo ello podemos concluir que la Educación Constitucional es un instrumento de transferencia a la sociedad civil del ODS $n^{\circ} 4$ al suponer la formación en los niveles obligatorios del sistema educativo de la Constitución y, con ello, en derechos fundamentales y en igualdad.

\section{La Educación Constitucional en el sistema constitucional español}

Traídos los planteamientos que venimos de analizar en los puntos anteriores al sistema constitucional español, la configuración de la Educación Constitucional dependerá -como

88 https://www.un.org/sustainabledevelopment/es/education/ Última consulta de todos los preceptos constitucionales que parecen citados en este trabajo: 18 de marzo de 2021. 
avanzamos en el epígrafe 1- de la compleja regulación constitucional de la materia educativa, la cual incidirá en las diferentes cuestiones problemáticas que a continuación examinamos.

\subsection{La Educación Constitucional en la Constitución de 1978}

La Constitución española de 1978, a diferencia de algunas de las constituciones de estados iberoamericanos ya mencionadas, no contiene ninguna previsión expresa sobre la enseñanza de la Constitución en los niveles obligatorios del sistema educativo (Educación Constitucional) o sobre la educación cívica.

Más en la línea de las constituciones sociales y normativas europeas, el texto fundamental español sí contiene un precepto específico sobre los fines de la acción educativa de los poderes públicos en el que aparece consagrado como fin "el pleno desarrollo de la personalidad humana en el respeto a los principios democráticos de convivencia y a los derechos y libertades fundamentales", precepto que recuerda que "el derecho a la educación se entiende como una de las manifestaciones más claras del desarrollo de la personalidad del individuo fundamento del orden jurídico y la paz social y, por ende, vinculado al concepto de dignidad humana " (Montero Caro, 2021: 6). No se refiere la Norma Fundamental explícitamente a que la Constitución haya de ser estudiada obligatoriamente en el sistema educativo, ni tan siquiera a que parte de sus contenidos esenciales -los principios democráticos y los derechos y libertades fundamentales que expresamente menciona- lo hayan de ser, sino a que todo el sistema educativo se enderezará a la consecución de estos tres valores de naturaleza política sobre los que se asienta el Estado constitucional para conseguir la limitación del poder político y de los que, de entrada, podemos afirmar que se refieren a la propia defensa del orden constitucional. Recordemos al respecto que la democracia y el respeto a los derechos humanos son dos de los tres principios que el movimiento constitucional insertó en las constituciones escritas para lograr con ellos la limitación del poder político del Estado; junto a ellos, el libre desarrollo de la personalidad, es decir, la libertad individual se estructuró -y estructura- como fin del propio Estado constitucional. La regulación del artículo 27.2 C.E. muestra, efectivamente, que "por lo que respecta a los fines, la Constitución ha querido ser beligerante en materia educativa" (Fernández Miranda, 2011: 255). 
Debe añadirse a lo anterior que, dado que el artículo 27.2 C.E. se incorpora a una Constitución en la que se contiene el explícito y sin ambages mandato de transformación social encomendado a los poderes públicos ex artículo 9.2 C.E., podemos traer aquí las conclusiones a las que arribamos en el punto 2 de este trabajo, las cuales nos permiten afirmar que, entre nosotros, el derecho a la educación está orientado a la consecución de estos fines como consecuencia de la general vocación transformadora de la sociedad encomendada a los podes públicos por este artículo 9.2 C.E. enderezada a asentar los valores del propio Estado Constitucional.

Estos planteamientos terminan desembocando en la existencia, en feliz expresión de Tomás y Valiente, de un "ideario educativo constitucional” que, siguiendo este punto las aportaciones de Aláez Corral, consiste en un "mandato a los poderes públicos y los particulares ordenándoles cuál debe ser el objetivo del derecho fundamental a la educación pública o privada, básica o superior, obligatoria o voluntaria” (Aláez Corral, 2011: 106).

Ideario constitucional que, en la interpretación de Aláez Corral, que es, asimismo, la nuestra, no "quiebra la necesaria neutralidad estatal en materia educativa puesto que esta se afirma [...] no con la finalidad de que el Estado se abstenga de inculcar a los individuos a través del sistema educativo las premisas epistemológicas y axiológicas de ese pluralismo democrático" (Aláez Corral, 2011: 109,110).

Como conclusión, en nuestra Constitución -a diferencia de otras- no existe expresamente la obligación de estudiar la Constitución, pero, al tiempo, existe un mandato según el cual la educación se enderezará a la consecución de los fines señalados como instrumento de defensa constitucional. Siendo ello así, el derecho a la educación del artículo 27.1 C.E. se ha de interpretar, en nuestro entendimiento, en el sentido de que, la "programación general de la enseñanza" (art. 27. 5 C.E.) en que consiste el objeto de esta enseñanza ha de estar orientada a la consecución de los fines anteriores. A partir de este entendimiento se plantea no solo la cuestión de (i) la posibilidad de que el legislador pueda introducir en la programación general de la enseñanza la Educación Constitucional, sino también una segunda sobre (ii) la existencia del mandato implícito al legislador en este sentido; y, también, (iii) una tercera sobre la cuestión de si el artículo 27.3 C.E. ampararía el derecho 
de los padres para que sus hijos reciban, como formación moral que esté de acuerdo con sus propias convicciones, la Educación Constitucional.

(i) Con respecto a la cuestión de la legitimidad de la Educación Constitucional en nuestro sistema ex artículo 27.2 C.E., la misma será constitucionalmente admisible siempre y cuando sus contenidos no sean contrarios a la libertad ideológica del alumnado; a la condición de democracia abierta de nuestro sistema; a la obligación de neutralidad de los poderes públicos; y a las libertades educativas reconocidas por la propia Constitución, en especial a la libertad de cátedra en su dimensión negativa y al derecho de los padres a recibir una formación moral o religiosa que esté de acuerdo con sus propias convicciones. Consiguientemente, el legislador podrá incorporar en la programación general de la enseñanza una Educación Constitucional fundamentada en el artículo 27.2 siempre que se respeten tales límites. A efectos expositivos nos referimos a la compatibilidad de la Educación Constitucional, en primer lugar, con la libertad de cátedra en su dimensión negativa, con la libertad ideológica del alumno y con la condición de democracia abierta de la nuestra; después, con el principio de neutralidad y con el derecho de los padres a recibir una formación moral o religiosa que esté de acuerdo con sus propias convicciones, ex artículo 27.3 C.E.

-Dado que, en la línea de lo expuesto con carácter general en los puntos 1 y 2 de este trabajo, el objeto de la Educación Constitucional es la explicación de la Constitución y solo de la Constitución, su fundamento es la defensa del orden constitucional y su función no es solo la de formar, sino también la de convencer, la legitimidad de este tipo de educación podría estar vedada en nuestro sistema como consecuencia de la prohibición, derivada de la libertad de cátedra en su dimensión negativa, de la existencia de una ciencia oficial; de la "prohibición de adoctrinamiento en razón de la libertad ideológica del alumno" (Cotino, 2012: 230); y como consecuencia de la propia condición de la democracia española de democracia abierta en la que, al no existir cláusulas pétreas o de intangibilidad, tiene cabida cualquier proyecto político, inclusive los que implican la ruptura del propio sistema constitucional.

En nuestra opinión, la Educación Constitucional se endereza no solo a la exposición de todos los valores y reglas de la Constitución, sino primordialmente a la exposición de la institución de la reforma constitucional y de su función como instrumento para el cambio 
político. Consiguientemente, enseñar la Constitución de 1978 significa, desde luego, formar en las instituciones políticas y en los derechos regulados en ella, pero también formar y convencer en que todo el sistema constitucional en vigor puede ser legalmente —y, por ello, legítimamente- alterado a través de la institución de la reforma constitucional, mecanismo con el que se logra la apoteosis y la sublimación del eterno motivo humano de que el Derecho someta al poder: gracias a la reforma constitucional, el cambio político se juridifica y, con ello y por primera vez en la Historia de la Humanidad, se convierte, además de en posible, en legítimo. Consecuentemente, la Educación Constitucional es un mecanismo de defensa de la reforma constitucional y, a través de ella, de la propia Constitución que, en última instancia, implica no solo convencer sobre la legitimidad de la Constitución, sino, sobre todo, convencer de que no existe una ciencia oficial, ni de que se proscribe cualquier proyecto diferente al previsto en la propia Constitución porque todos los valores y reglas de la Constitución pueden ser alterados respetando los mecanismos que para ello prevé la propia Constitución a través de su Título X. Consiguientemente, la formación del alumnado en una Constitución sin cláusulas pétreas y con un procedimiento de reforma constitucional que, precisamente por ello, permite la modificación de todos sus contenidos, sin excepción, no implica la imposición de una ciencia oficial, ni conculca la libertad ideológica del alumnado ni tampoco impone la prohibición de perseguir y lograr proyectos políticos que, aunque no estén incorporados a la Constitución vigente, pueden serlo a través del instituto de la reforma constitucional.

Por el contrario, la prohibición de adoctrinamiento fundamentada en la libertad ideológica del alumnado, en la dimensión negativa de la libertad de cátedra y en el principio de neutralidad de los poderes públicos sí impediría la impartición en los niveles obligatorios del sistema educativo toda educación para la convivencia que exceda de los contenidos directa e inmediatamente incardinados en la Norma Fundamental. Siendo ello así, una Educación para la Ciudadanía solo puede existir identificada como Educación Constitucional, es decir, solo es legítima una educación en valores o una educación para la ciudadanía si la misma se limita a la explicación de la Constitución, es decir, si la misma se identifica con la Educación Constitucional.

-Una vez asumida que la dimensión negativa de la libertad de cátedra y la libertad ideológica del alumnado no impediría una Educación Constitucional, cabe plantearse, a 
partir de ello, si todas las reglas y valores de la Constitución podrían incluirse en la Educación Constitucional o si, por el contrario, dado el pluralismo político que aparece consagrado como valor superior de nuestro ordenamiento jurídico y el principio de neutralidad ideológica del Estado español, los contenidos de la Educación Constitucional han de limitarse tan solo a aquellos desarrollos constitucionales que no han quedado a la libre decisión de las sucesivas mayorías sucesivas (dialéctica política) como consecuencia de que sea la propia Constitución la que se abra a posibilidades, constitucionalmente legítimas, pero contradictorias.

En nuestra opinión, la Educación Constitucional ha de limitarse a los contenidos que se deducen necesariamente de la Norma Fundamental. Esta es la postura a la que ha llegado el Tribunal Supremo, no con respecto a la Educación Constitucional, pues la misma como veremos- no se ha incorporado a nuestro sistema educativo, sino a propósito de la Educación para la Ciudadanía y los Derechos Humanos que, entre sus varios contenidos, presentaba como uno de ellos la explicación parcial de la Constitución. Con respecto a ellos, el Tribunal Supremo ha considerado que es lícita la actividad educativa referente a "los valores necesarios para el buen funcionamiento del servicio democrático" y el "entramado institucional del Estado" (STS 342/2009, de 11 de febrero). Con ello, el Tribunal Supremo considera como legítima en nuestro sistema la Educación Constitucional siempre que se limite a los contenidos directa e inmediatamente derivados de la Constitución. Consecuentemente, según el Tribunal Supremo una Educación Constitucional es legítima en tanto en cuanto respete el principio de neutralidad y el principio de pluralismo político, lo que impone su constricción a la exposición de los contenidos constitucionales anteriores.

Delimitado así el contenido de la Educación Constitucional, frente a la misma no puede invocarse el derecho de los padres a elegir la formación moral y religiosa de los hijos, el cual, efectivamente, no alcanza a "cualquier tipo de prohibición de transmisión de conocimientos científicos o a la enseñanza de los principios constitucionales -los propios del pluralismo político e ideológico- y de los que fundamentan una sociedad democrática" (González-Varas, 2015: 121).

A resultas de lo expuesto hasta aquí, podemos concluir que el artículo 27.2 C.E. legitima la existencia de la Educación Constitucional, siempre y cuando la misma se limite -en 
palabras del Tribunal Supremo- a "los valores necesarios para el buen funcionamiento del servicio democrático" y al "entramado institucional del Estado": de igual modo, el artículo 27.2 C.E. no avalaría una Educación Cívica de contenidos sociales que no se hallan en la Norma Fundamental.

(ii) ¿Cabe deducir de la regulación de los artículos 9.2 C.E. y 27.2 C.E., en relación con el 27.1 C.E. y el 27.5 C.E. la existencia del mandato implícito de estudiar, al menos, "los principios democráticos de convivencia y a los derechos y libertades fundamentales" de forma que la programación general de la enseñanza, objeto del derecho a la educación, ha de prever obligatoriamente la enseñanza de los principios anteriores?

En nuestra opinión, tal mandato implícito existe a partir de la lectura del art. 27.2 C.E. a la luz del 9.2 C.E. e, incluso, de la cláusula preambular "sociedad democrática avanzada"; tal mandato obligaría al legislador a introducir la Educación Constitucional configurada tal como venimos de hacer.

Como hemos adelantado, el derecho a la educación recogido en el artículo 27.1 C.E. supone la garantía de la efectividad del acceso al saber incluyendo como uno de sus contenidos el acceso a las enseñanzas del sistema educativo, las cuales estarán orientadas a la consecución de los fines del artículo 27.2 C.E. Puede considerarse que tales fines se satisfarían con una organización de la educación que ha de ser democrática y garantista de los derechos humanos, sin que impliquen la introducción en la programación general de enseñanza de la Educación Constitucional; sin embargo, a la conclusión anterior cabe objetar que el 27.1 C.E. y el 27.2 C.E. han de interpretarse a la luz del artículo 9.2 de la Constitución que consagra que los poderes públicos han de "promover las condiciones para que la libertad y la igualdad del individuo y de los grupos en que se integra sean reales y efectivas", es decir, han de orientarse a la consecución de una transformación de la sociedad enderezada a la consecución de los valores superiores del ordenamiento jurídico, transformación que se logra primordialmente mediante una adecuada educación constitucional en la que se forme a la ciudadanía tales valores. Añádase a ello la exigencia interpretativa que la cláusula preambular "sociedad democrática avanzada" impone en ese sentido y que determina que, precisamente, construir una sociedad democrática avanzada implica profundizar en una transformación social enderezada a la consecución 
de los valores superiores que recoge la Constitución, para lo cual se revela como imprescindible una adecuada educación constitucional.

(iii) Partiendo de que los principios del artículo 27.2 C.E. no reconocen derechos fundamentales (STC 5/1981), cabría plantearse si tendría lugar en nuestro sistema un planteamiento según el cual la enseñanza de la Constitución sería el objeto del derecho fundamental a recibir una adecuada educación constitucional en la enseñanza obligatoria, derecho de libertad integrado en el art. 27.3 C.E. consistente en el derecho que asiste a los padres para que sus hijos reciban la formación religiosa y moral que esté de acuerdo con sus propias convicciones, en este caso la formación moral centrada en los valores superiores del ordenamiento jurídico: la libertad, la justicia, la igualdad y el pluralismo político, que son los propios del constitucionalismo.

\subsection{La Educación Constitucional en la legislación en materia educativa}

En cualquier caso, partiendo de la centralidad del artículo 27.2 y más allá de que “existen discrepancias importantes sobre el concepto y la intensidad del objeto de la educación determinado en el artículo 27.2" (Vidal Prado, 2017:55), el legislador español ha introducido entre nosotros a la Educación Constitucional, la cual, si bien no con esta denominación, fue tempranamente regulada por la Ley 19/1979, de 3 de octubre, por la que se regula el conocimiento del ordenamiento constitucional en Bachillerato y en Formación Profesional de primer grado, ley que disponía en su artículo 1 que "A partir del año académico mil novecientos setenta y nueve se incluirá, entre las enseñanzas comunes en los Planes de Estudio de Bachillerato y de Formación Profesional de Primer Grado, el Conocimiento del Ordenamiento Constitucional en general y su desarrollo estatutario en las nacionalidades y regiones que tengan aprobado Estatuto de Autonomía". Esta ley identificaba en su artículo 3 el objeto de la Educación Constitucional señalando que "el contenido de estas enseñanzas se orientará fundamentalmente a proporcionar a los alumnos una información suficiente de los derechos y libertades que integran la Constitución Española, así como los contenidos en los Tratados y Convenios Internacionales ratificados por España; la organización del poder en el Estado español y su estructuración territorial”. 
La posterior Ley Orgánica 8/1985, de 3 de julio, reguladora del Derecho a la Educación elevó el conocimiento del ordenamiento constitucional a derecho-deber de los educandos al establecer expresamente en su número dos que "Todos los alumnos y alumnas tienen el derecho y el deber de conocer la Constitución Española y el respectivo Estatuto de Autonomía, con el fin de formarse en los valores y principios reconocidos en ellos". Su objeto concreto, en los términos del artículo sexto de la Ley Orgánica 8/1985, de 3 de julio, reguladora del Derecho a la Educación, es “[...] conocer la Constitución Española y el respectivo Estatuto de Autonomía, con el fin de formarse en los valores y principios reconocidos en ellos".

Bajo la vigencia de la LODE, la Educación Constitucional nunca ha sido objeto de una asignatura autónoma específicamente dedicada a la misma; en todo caso, algunos contenidos de la Constitución han constituido uno de los objetos de otras asignaturas autónomas similares que, por venir centradas en el estudio de la sociedad o de la convivencia en sociedad, debían referirse a la Constitución. Este es el supuesto de la asignatura Educación para la Ciudadanía y los Derechos Humanos (en adelante, EPC) entre cuyos contenidos figuraba, precisamente, la explicación de las libertades públicas (art. 18.3 de la L.O. 2/2006, de 3 de mayo, de Educación). Asimismo, este es el supuesto de la actual asignatura alternativa a la de Religión en la Educación Primaria, la “Educación en valores sociales y cívicos" (art. 8 del Real Decreto 126/2014, de 28 de febrero, por el que se establece el currículo básico de la Educación Primaria), y de la nueva "Educación en valores cívicos y éticos" introducidos por la Ley Orgánica 3/2020, de 29 de diciembre, por la que se modifica la Ley Orgánica 2/2006, de 3 de mayo, de Educación, la cual se impartirá en uno de los cursos del último ciclo de la Educación Primaria (quinto o sexto) y en otro curso de los seis cursos de la Educación Secundaria y en la que se incluirán no solo "contenidos referidos a la Constitución española", sino también "al conocimiento y respeto de los Derechos Humanos y de la Infancia, a la educación para el desarrollo sostenible y la ciudadanía mundial, a la igualdad entre hombres y mujeres, al valor del respeto a la diversidad y al valor social de los impuestos, fomentando el espíritu crítico y la cultura de paz y no violencia”.

La Educación Constitucional tampoco se ha configurado como un elemento transversal de los niveles obligatorios del sistema educativo, si bien el conocimiento de algunos de los contenidos de la Constitución sí lo ha sido, como ponía de manifiesto la configuración 
de la "educación en valores" en el derogado artículo 18.3 de la L.O. 2/2006, de 3 de mayo, de Educación, según el cual "Sin perjuicio de su tratamiento específico en algunas de las áreas de la etapa, la comprensión lectora, la expresión oral y escrita, la comunicación audiovisual, las Tecnologías de la Información y la Comunicación, el emprendimiento y la educación cívica y constitucional se trabajarán en todas las áreas”. En desarrollo de este precepto, el artículo 6.2 del Real Decreto 1105/2014, de 26 de diciembre, por el que se establece el currículo básico de la Educación Secundaria Obligatoria y del Bachillerato -todavía en vigor- regula los "elementos transversales" disponiendo al respecto que "las Administraciones educativas fomentarán el desarrollo [...] los valores inherentes al principio de igualdad de trato y no discriminación por cualquier condición o circunstancia personal o social; asimismo, las Administraciones educativas fomentarán el aprendizaje [...] de los valores que sustentan la libertad, la justicia, la igualdad, el pluralismo político, la paz, la democracia, el respeto a los derechos humanos, el respeto a los hombre y mujeres por igual, a las personas con discapacidad y el rechazo a la violencia terrorista, la pluralidad, el respeto al Estado de derecho [...]”.

Consiguientemente, la legislación educativa configura a la Educación Constitucional como derecho/deber del alumnado de Educación Primaria y de Educación Secundaria, pero, al mismo tiempo, los currículos básicos de Educación Primaria y de Educación Secundaria garantizan solo el conocimiento de aspectos parciales de la Constitución y, además, o bien como elementos transversales de los niveles obligatorios del sistema educativo, o bien como uno de los contenidos de una asignatura alternativa a la de Religión en la Educación Primaria (en la regulación en vigor hasta la aprobación Ley Orgánica 3/2020, de 29 de diciembre), o bien como uno de los contenidos de una asignatura obligatoria para todo el alumnado, pero centrada en los Valores cívicos y sociales y, por ello, no identificable plenamente con la Educación Constitucional (a partir de la aprobación Ley Orgánica 3/2020, de 29 de diciembre).

Mención aparte merece la problemática que suscita la formación en valores constitucionales en el ámbito de los centros docentes militares. Por su configuración, la Educación Constitucional no existe propiamente en este ámbito, dado que esta no es sino la formación en la Constitución del alumnado de los niveles obligatorios del sistema educativo, y no en el ámbito de las relaciones de especial sujeción que representa la institución militar. Concretamente, en los centros docentes militares no existe una 
educación formal de su alumnado centrada en la Constitución que pudiéramos considerar como trasunto en el ámbito castrense de la Educación Constitucional.

No obstante ello, sí existe una educación no-formal de la Constitución en el ámbito de la enseñanza de formación de las Fuerzas Armadas, es decir, de la impartida en un centro docente militar de formación o centro universitario de la Defensa con la finalidad de proporcionar la preparación y capacitación para el ejercicio profesional y la incorporación o adscripción a los diferentes cuerpos, escalas y la obtención de especialidades. En este sentido, la reciente Instrucción 2/2021, de 8 de enero, de la Subsecretaria de Defensa, por la que se establecen normas sobre determinadas actividades de régimen interior en los centros docentes militares para el fomento de los principios y valores constitucionales, establece que cualquier acto, sea de la naturaleza que fuere (órdenes diarias, discursos, alocuciones, charlas de motivación, conferencias, prácticas o cualquier otra llevada a cabo en grupo por y para los alumnos) que se realice en un centro militar deberá estar en consonancia con los principios establecidos por la Constitución Española, especialmente en lo relacionado con la convivencia en democracia y el respeto a los valores que la inspiran. Asimismo, esta Instrucción dispone que en tales actos se habrá de evitar cualquier uso o exhibición de simbología que vulnere las normas, los principios y valores constitucionales. Tal Instrucción se dicta, por su parte, en aplicación del artículo 6 del Real Decreto 1051/2020, de 1 de diciembre, por el que se aprueba el Reglamento de ordenación de la enseñanza de formación en las Fuerzas Armadas, el cual dispone que la enseñanza de formación se regirá por -entre otros- el principio de fomentar los principios y valores constitucionales (la pluralidad cultural de España, el respeto a los derechos y libertades fundamentales, la igualdad de derechos y oportunidades entre hombres y mujeres, la protección del medio ambiente, la no discriminación de las personas por razón de nacimiento, raza, sexo, religión, opinión o cualquier otra condición o circunstancia personal o social, el respeto a la justicia y el ejercicio de la tolerancia y de la libertad dentro de los principios democráticos de convivencia).

\section{Conclusiones}

-La Educación Constitucional es la formación en los valores y reglas constitucionales en los niveles obligatorios del sistema educativo. Su objeto científico es la Constitución y no la convivencia o la vida en común; consiguientemente se diferencia de asignaturas 
como "Valores éticos o sociales" o "Educación ciudadana". Su destinatario, además, es el alumnado de los niveles obligatorios del nivel educativo, y no el alumnado de la Educación Superior, diferenciándose, por ello, del Derecho Constitucional.

-La denominación "Educación Constitucional" no ha adquirido hasta el momento carta de naturaleza, si bien la obligatoriedad del estudio de la Constitución de un Estado en los niveles obligatorios de su sistema educativo se recoge en varias constituciones, tanto históricas, la española de 1812 y la alemana de 1919, como actuales, todas ellas de estados iberoamericanos: Colombia; El Salvador de 1983; Guatemala; Honduras; Nicaragua de 1987; Paraguay; Perú; y, por último, República Dominicana.

-El fundamento de la Educación Constitucional no se encuentra en exigencias del sistema educativo, sino en la defensa del orden constitucional, en el entendido de que las constituciones han de ser conocidas por quienes están llamados a vivir bajo ellas. Tal fundamento se halla en el análisis de las constituciones de Cádiz y de Weimar, las primeras que, en los albores de los constitucionalismos liberal y social, respectivamente, incorporaron la enseñanza de la Constitución entre sus preceptos.

-La educación para los derechos humanos y la educación para la igualdad son, por definición, dos contenidos de la Educación Constitucional, pues los derechos y la igualdad ya no son solo dos de los contenidos típicos de toda Constitución, sino dos de los principios esenciales del constitucionalismo que, asimismo, aparecen en la Historia vinculados a la ideología constitucional. Consiguientemente, la Educación Constitucional es un instrumento de transferencia a la sociedad civil del ODS $\mathrm{n}^{\circ} 4$ al suponer la formación en los niveles obligatorios del sistema educativo de la Constitución y, con ello, en derechos fundamentales y en igualdad.

-En el sistema constitucional español, encontramos el artículo 27.1 C.E. que consagra el derecho a la educación como derecho fundamental de carácter prestacional orientado a los fines que establece el artículo 27.2 C.E., que son el pleno desarrollo de la personalidad humana en el respeto a los principios democráticos de convivencia y a los derechos y libertades fundamentales. Estos fines del derecho a la educación forman parte de la general vocación transformadora de la sociedad orientada a la consecución de los valores constitucionales de justicia, libertad igualdad y pluralismo político encomendada a los 
poderes públicos por el artículo 9.2. C.E. Junto con el derecho a la educación, también encontramos la libertad ideológica del alumno; la prohibición de ciencia oficial, derivada de la libertad de cátedra en su dimensión negativa; el principio de neutralidad de los poderes públicos; y el derecho de los padres para que sus hijos reciban una formación religiosa y moral que esté de acuerdo con sus propias convicciones. Del juego de estos preceptos llegamos a las siguientes conclusiones: a) La Educación Constitucional es legítima en el ordenamiento español, siempre y cuando se circunscriba a "los valores necesarios para el buen funcionamiento del servicio democrático" y el "entramado institucional del Estado" (STS 342/2009, de 11 de febrero), entre los cuales figura la institución de la reforma constitucional, mediante la cual todo cambio político es posible y legítimo; b) La Educación Constitucional, además, es obligada en el ordenamiento constitucional, siempre y cuando se configure dentro de los límites precisos anteriormente señalados.

-La legislación educativa configura a la Educación Constitucional como derecho/deber del alumnado de Educación Primaria y de Educación Secundaria, pero, al mismo tiempo, los currículos básicos de Educación Primaria y de Educación Secundaria la desconocen. Efectivamente, tales currículos no disponen la obligatoriedad de la enseñanza de la Constitución, sino tan sólo de algunos de sus contenidos y, en modo alguno, de todos los posibles. Además, tales contenidos se organizan, bien como elementos transversales de los niveles obligatorios del sistema educativo, bien como uno de los contenidos de una asignatura alternativa a la de Religión en la Educación Primaria, bien como uno de los contenidos de una asignatura obligatoria para todo el alumnado, pero centrada en los Valores cívicos y sociales.

\section{Bibliografía}

ALÁEZ CORRAL, B. (2011). El ideario educativo constitucional como límite a las libertades educativas. Revista Europea de Derechos Fundamentales. 91-129.

ARGÜELLES, A. (2011). Discurso preliminar a la Constitución de 1812. Introducción de L. Sánchez Agesta. Madrid: CEPC. 
COTINO HUESO, L. (2012). El derecho a la educación como derecho fundamental. Especial atención a su dimensión social prestacional. Madrid: Centro de Estudios Políticos y Constitucionales.

FERNÁNDEZ-MIRANDA CAMPOAMOR, A. (2011). "Derecho a la educación”. En C. Aguado Renedo; M. Aragón Reyes. Temas Básicos de Derecho Constitucional. Volumen III. Madrid: Thomson Reuters (pp. 254-249).

GARCÍA COSTA, F.M. (2019). "La defensa paidética de la Constitución española de 1812”. En F.M. García Costa et altri (Co). Historia Constitucional de Iberoamérica. Valencia: Tirant lo Blanch (pp. 345-396).

GARRORENA MORALES, A. (2011). Derecho Constitucional. Teoría de la Constitución y Sistema de Fuentes. Madrid: CEPC.

GONZÁLEZ-VARAS IBÁÑEZ, A. (2015). Derechos educativos, calidad en la enseñanza y proyección jurídica de los valores en las aulas. Valencia: Tirant lo Blanch.

MONTERO CARO, M. D. (2021). Educación, Gobierno Abierto y progreso: los Objetivos de Desarrollo Sostenible (ODS) en el ámbito educativo. Una visión crítica de la LOMLOE. Revista de Educación y Derecho, 23, 1-26.

ONU: https://www.un.org/sustainabledevelopment/es/education/

Proyecto Constitute https://www.constituteproject.org

VIDAL PRADO, C. (2017). El derecho a la educación en España: bases constitucionales para el acuerdo y cuestiones comprometidas, Madrid: Marcial Pons. 